\title{
Heterodyne measurement of parametric dispersion in electromagnetically induced transparency
}

\author{
M. Müller, F. Homann, R.-H. Rinkleff, and A. Wicht \\ Institut für Atom- und Molekülphysik, Abteilung Spektroskopie, Universität Hannover, Callinstraße 38, D-30167 Hannover, Germany \\ K. Danzmann \\ Institut für Atom- und Molekülphysik, Abteilung Spektroskopie, Universität Hannover, Callinstraße 38, D-30167 Hannover, Germany \\ and Max-Planck-Institut für Quantenoptik, Außenstelle Hannover, Callinstraße 38, D-30167 Hannover, Germany
}

(Received 07 December 2000; published 29 May 2001)

\begin{abstract}
Using three phase-locked lasers, we have observed in a three-level system the phase shift of the frequency fixed coupling laser in dependence of the probe laser frequency relative to an off-resonant reference laser. In the vicinity of the two-photon resonance, the system exhibits electomagnetically induced transparency and we show that the dispersion of coupling and probe field shows different spectra if the probe field frequency is tuned. The parametric dispersion of the coupling field is measured on a cesium atomic beam with a heterodyne interferometer over several decades of coupling and probe laser power. Even with coupling powers in the $\mathrm{nW}$ range, the interferometer gives a good signal-to-noise ratio. The results are in good agreement with a numerical simulation of a semiclassical model. Analytical expressions for the dispersion are given.
\end{abstract}

DOI: 10.1103/PhysRevA.64.013803 PACS number(s): 42.50.Gy, 32.70.Jz, 07.57.Pt, 07.57.Ty

\section{INTRODUCTION}

Coherently driven atomic systems have been studied extensively during the past few decades. The application of coherent laser fields onto atomic systems consisting of a number of states leads to astonishing new effects and the realization of new types of media. One of the most impressive effects that can occur is electromagnetically induced transparency (EIT): An opaque medium becomes transparent for a probe laser field in the presence of a coupling field. Even if it is most unlikely to make laser beams propagate through walls [1], EIT has been shown to occur in solid materials [2].

Likewise, the dispersive properties can be designed. Strong positive and negative dispersion can be created as well as a high refractive index [3] or large Kerr nonlinearities [4]. Negative dispersive transparent media can be used to realize an optical cavity with large buildup but broadband response (white light cavity) $[5,6]$ or to demonstrate superluminal light propagation [7]. Strong positive dispersion with simultaneously vanishing absorption has been used to slow electromagnetic pulses down to extremely low velocities of several meters per second [8]. This large reduction is typically accompanied by an enhancement of the Kerr nonlinearity. The Kerr nonlinearity is given by the ratio of the coherence time of the forbidden ground-state transition to the spontaneous-emission time of the alkali-metal atoms used in this demonstration [9]. Scully and co-workers have shown that slow light can enhance the acousto-optical effect and promised applications in quantum optics such as squeezing and quantum nondemolition measurements [10]. Positive dispersive media have also found applications as ultrasensitive magnetometers $[11,12]$ and as a setup of a low finesse but narrow linewidth optical cavity [13].

A system often used to create EIT is the $\Lambda$-type threelevel system. Two laser fields are coupling two states to a common excited level. No electromagnetic dipole transitions are allowed between the two lower states. This system was first studied experimentally in 1976 by Alzetta et al. [14]. Within a relatively broad absorption (several $\mathrm{MHz}$ for alkalimetal vapors), there is an extremely narrow transparency window (down to $100 \mathrm{~Hz}$ and less) for both coupling and probe field due to destructive quantum interference of the two transition probability amplitudes.

The dispersive properties of this dark resonance have been studied experimentally focusing on the probe field dispersion [15] or on the relative phase shift between coupling and probe field [16]. But even in this well-known system, surprising effects can still be found. While the probe field shows a broad dispersion line with a narrow inverted dispersion curve in the center, the coupling field only shows a single narrow dispersive feature at the two-photon resonance. We call this behavior parametric dispersion [17]. In this work, we will give a detailed overview of the interferometric method we used to measure the parametric phase shift and the semiclassical model used to describe the phenomenon.

The parametric dispersion can be seen as a cross correlation between the frequency of the probe field and the phase of the coupling field. On the EIT resonance, it is therefore possible to realize a lossless opto-optical phase modulator or an opto-optical deflector for the coupling beam.

\section{EXPERIMENT}

To realize electromagnetic induced transparency, we are using a $\Lambda$-type three-level system in the $D_{2}$ line $(852 \mathrm{~nm})$ of ${ }^{133} \mathrm{Cs}$ (see Fig. 1). The two hyperfine levels of the ground state are coupled to a common hyperfine level of the excited state via a coupling and a probe field. The phase shift of the coupling field is measured with a three-beam heterodyne interferometer relative to a reference field. The coupling and the probe field prepare the atoms in a coherent superposition of the two ground states and the reference field is far off- 


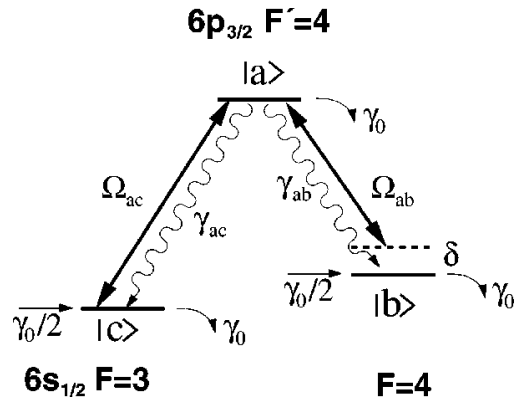

FIG. 1. $\Lambda$-type three-level system. $|i\rangle$ : eigenstates of the unperturbed Hamiltonian; $\gamma_{a b}, \gamma_{a c}$ : spontaneous emission rates; $\gamma_{0} / 2$ : injection rate; $\gamma_{0}$ : transient loss.

resonance from the optical transitions. A simple example for a heterodyne interferometer without an additional reference field is described in [16]. The advantage of our setup is that the dispersion of both probe and coupling field can be measured independently (and simultaneously).

Compared to other quasiheterodyne techniques such as frequency modulation spectroscopy, we can use much higher frequency offsets for the reference field than is possible to achieve (efficiently) with electro-optic phase modulators. A high-frequency offset avoids unwanted interaction of the reference field with the sample. In our experiment, we have chosen the frequency offset high enough in comparison to the hyperfine splitting of the excited level and small enough in comparison to the hyperfine splitting of the ground level $(9.2 \mathrm{GHz})$ so that no interaction with the atoms was expected. In addition, the use of a separate laser field offers more flexibility to choose frequency, polarization, and intensity.

Standard homodyne techniques such as the Mach-Zehnder interferometer require very good mechanical stability of the optical setup. Each change in the arm lengths due to acoustic or seismic noise produces an output signal that is indistinguishable from the atomic response. In the heterodyne interferometer, this noise only contributes on the scale of the frequency offset of the reference field (which is in the microwave range) rather than on the scale of an optical frequency. For a detailed critical survey of the competitive methods, i.e., with interferometry and frequency modulation spectroscopy, the reader is referred to the work of Wicht et al. [18].

\section{SETUP}

A schematic of the setup is given in Fig. 2. The heterodyne interferometer consists of the coupling laser, the probe laser, and the off-resonant reference laser. The lasers are all single-mode diode lasers, passively stabilized by a Hollbergtype setup. The linewidth of the lasers is $100 \mathrm{kHz}$ at time scales of milliseconds. The coupling laser drives the hyperfine transition $6 s_{1 / 2}, F=3 \rightarrow 6 p_{3 / 2}, F^{\prime}=4$. The frequency is actively locked with an error signal generated by a Dopplerfree polarization spectroscopy. The probe laser frequency is scanned around the $6 s_{1 / 2}, F=4 \rightarrow 6 p_{3 / 2}, F^{\prime}=4$ transition.

In order to achieve maximum coherence effects, the three fields are phase-stabilized in a chain of two phase-locked loops. The difference frequency $d \omega_{\text {ref }} /(2 \pi) \approx 1 \mathrm{GHz}$ between coupling and reference field is provided by an ultrastable tuneable microwave synthesizer (Rhode\&Schwartz, SMT06). All fluctuations of the laser difference frequency are eliminated by the phase-locked loop (PLL). Care was taken that the reference laser field did not interact with the atomic sample. The second PLL locks the probe field to the reference field with a difference frequency of $d \omega_{\text {probe }}$

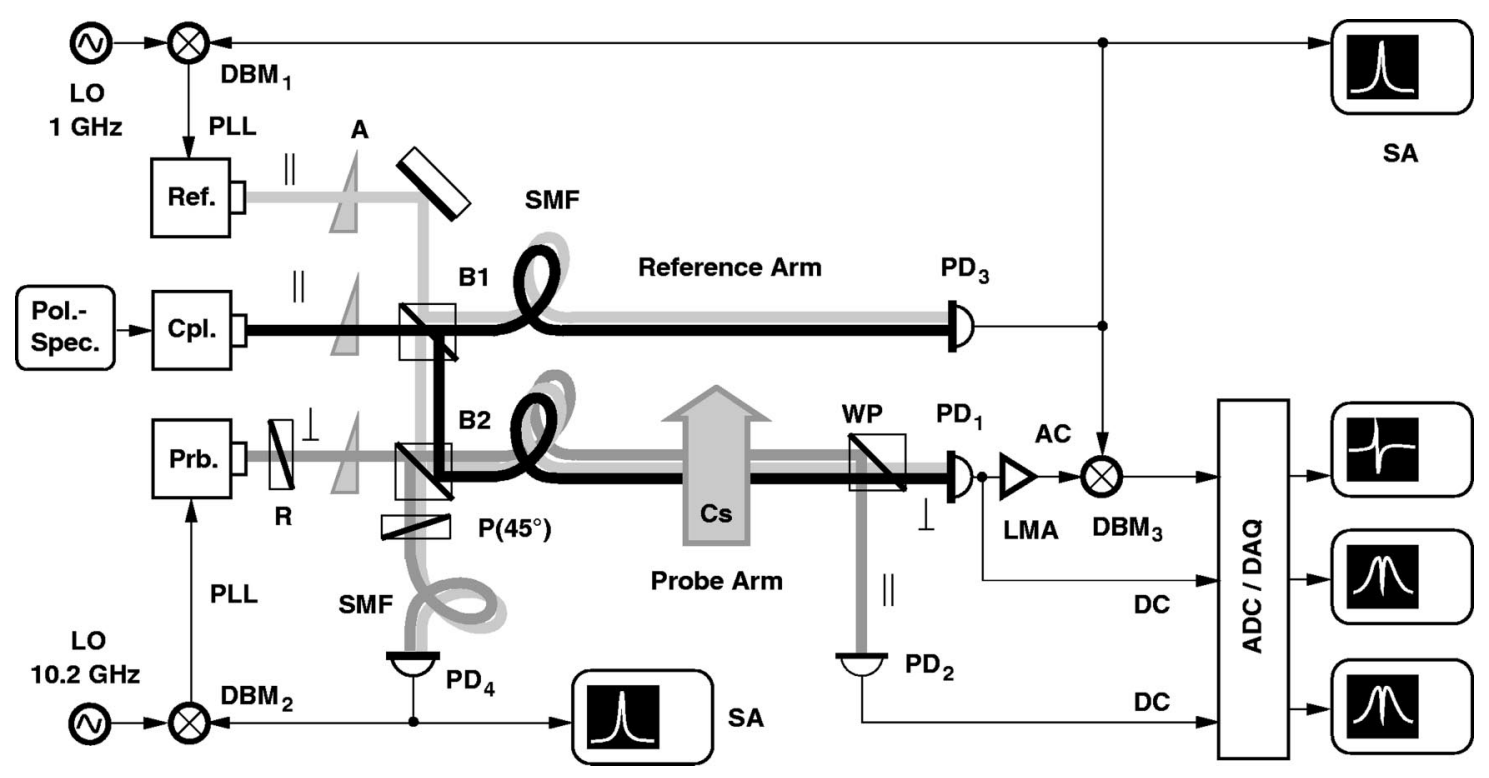

FIG. 2. Experimental setup. Prb., Cpl. and Ref.: probe, coupling, and reference laser; $\|$ : parallel polarization; $\perp$ : vertical polarization; B1,B2: power beam splitters; $P$ : polarizer; $P\left(45^{\circ}\right)$ : polarizer under $45^{\circ}$ with respect to the incident polarization; R: $\lambda / 2$ retarder; $W P$ : Wollaston prism; Cs: cesium atomic beam; SMF: single-mode fiber; PD: photodiodes; LO: local oscillator; SA: spectrum analyzer; DBM: double balanced mixer; LMA: limiting amplifier; ADC/DAQ: analog-digital converter/data acquisition; PLL: optical phase-locked loop. 

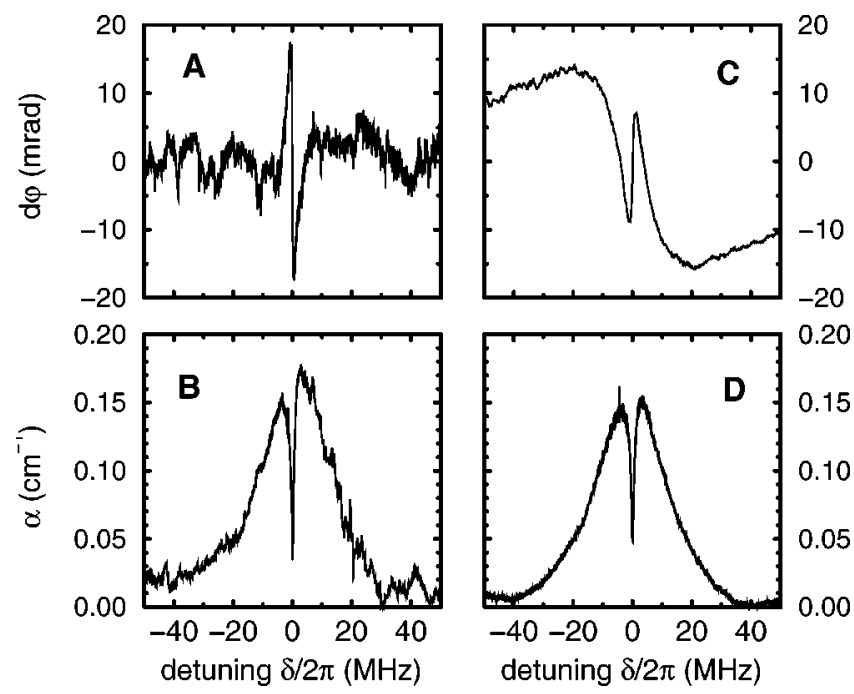

FIG. 3. Typical observed spectra of the parametric phase shift $d \varphi_{\text {coupl }}(A)$ and the corresponding absorption $\alpha(B)$. In $C$ and $D$, phase shift and absorption of the probe field are shown. Probe and coupling laser intensity were $2.5 \mathrm{~mW} / \mathrm{cm}^{2}$.

$=d \omega_{\text {ref }}+\delta+2 \pi 9.19263177 \mathrm{GHz}$, where $\delta$ is the twophoton detuning. The difference frequency is generated by a second tuneable microwave synthesizer (Rhode\&Schwartz, SMP02). When all lasers are phase-locked, the beat signals between any two lasers cannot be resolved within a resolution bandwidth of $3 \mathrm{~Hz}$ on the spectrum analyzer. The residual phase noise of the PLL is $10^{-4} \mathrm{rad} / \sqrt{\mathrm{Hz}}$. For a detailed description of the technique of optical phase locking, the reader is referred to [19].

Coupling and reference field are superposed at the beam splitter B1. The beam splitter divides the fields into the reference and probe arm of the heterodyne interferometer. At B2, the probe field is superposed. One output continues into the probe arm while the other output is used to detect the beat signal between the probe and reference field. This beat signal is detected with a fiber-coupled $25-\mathrm{GHz}$ bandwidth $p-i-n$ photodiode $\left(\mathrm{PD}_{4}\right.$, New Focus 1431) and observed on a Tektronix 2782 spectrum analyzer. The signal is demodulated at $d \omega_{\text {probe }}$ with a double-balanced microwave mixer $\left(\mathrm{DBM}_{2}\right.$, Miteq M0812). The intermediate frequency output provides the error signal for the PLL between the reference and probe field. The beat signal between the reference and coupling field is detected with a similar $p-i$ - $n$ photodiode at the end of the reference arm $\left(\mathrm{PD}_{3}\right)$. The signal is split, and one part is mixed with a local oscillator frequency at $d \omega_{\text {ref }}$ $\left(\mathrm{DBM}_{1}\right.$, Miteq DM0052LA2). The mixer output is the error signal for the PLL. The frequency of the beat signal is observed with another microwave spectrum analyzer (HP8562E).

The reference and coupling field are horizontally polarized and the probe field is polarized vertically. After the interaction zone, the probe beam is separated from the other fields with a Wollaston prism (extinction better than $10^{-5}$ ). The beat between reference and coupling field is detected with a $1-\mathrm{GHz}$ bandwidth photoreceiver $\left(\mathrm{PD}_{1}\right.$, New Focus 1601). The signal is amplified to a constant level by a limiting microwave amplifier (LMA, Miteq AMF-5F009011-3010P-LM) and demodulated with the signal from the reference arm with a microwave mixer $\left(\mathrm{DBM}_{3}\right.$, Miteq DM0052LA2).

To achieve good mode matching in the probe arm, the three fields are guided through a single-mode fiber (New Focus 1234). After the fiber, the fields are collimated with an achromatic lens doublet that produces a waist in the interaction zone of $w=2200 \mu \mathrm{m}$. The intensity distribution after the collimator correlates to $97 \%$ with a Gaussian profile, measured with a Melles-Griot beam analyzer. The intensity of the fields is controlled by metallic neutral density filters. The filters are mounted on stable wheels to get good reproducibility.

A continuous flow reflux oven [20] produces a beam of cesium atoms with a collimation ratio of 1:50. The rest Doppler width of the optical transition and of the two-photon resonance is $4.5 \mathrm{MHz}$ and $120 \mathrm{~Hz}$, respectively. At $180^{\circ} \mathrm{C}$, the atomic density is $8 \times 10^{8} \mathrm{~cm}^{-3}$, and from the Maxwell distribution law we derive the most probable velocity of the atoms, $v=240 \mathrm{~m} / \mathrm{s}$. The average transient time through the 2.2-mm waist of the laser beam is then $\tau=w / v \approx 10^{-5} \mathrm{~s}$. The width of the atomic beam and therefore the length of the interaction region is $5 \mathrm{~mm}$. An additional nitrogen-cooled aperture ensures a low background of cesium gas in the ultrahigh-vacuum chamber $\left(<10^{-8} \mathrm{mbar}\right)$. Six coils in the three spatial directions, in the Helmholtz configuration, sup-
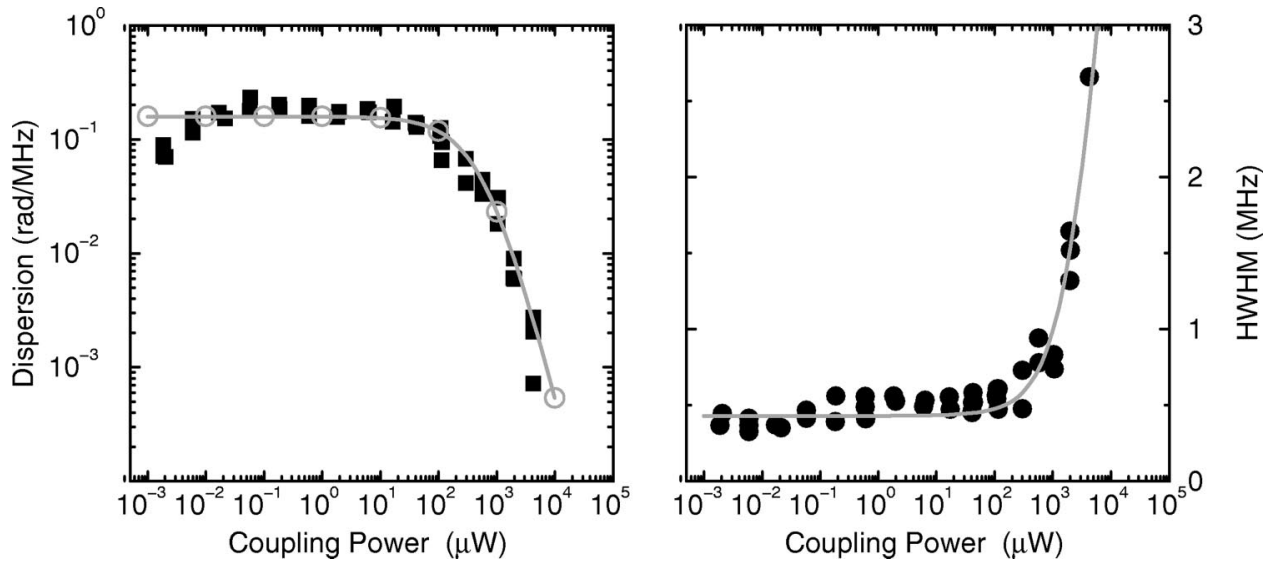

FIG. 4. Left: Absolute value of the observed parametric phase shift of the coupling field with probe laser intensity $3.1 \mathrm{~mW} / \mathrm{cm}^{2}$. The solid curve shows the semiclassical solution from Eq. (A10). Right: Linewidth of the dispersion curve. The circles show the approximated solution for $\gamma_{0}$ $=10^{5} \mathrm{~s}^{-1} \ll \gamma$ from Eq. (5.3). HWHM: half-width at half maximum. 
press unwanted magnetic fields in the interaction region below $5 \mathrm{mG}$.

\section{SIGNALS}

While phases and amplitudes in the reference arm remain constant, the phase of the coupling field in the probe arm is shifted by $d \varphi_{\text {coupl }}(\delta)$. This phase shift depends on the probe detuning $\delta$. The beat signals of the probe and reference arm are electronically multiplied by the mixer $\mathrm{DBM}_{3}$. The mixer output signal then provides the desired phase information:

$$
I_{\text {mix }}(\delta) \propto\left(E_{0}^{\text {coupl }} E_{0}^{\mathrm{ref}}\right)^{2} e^{-\alpha(\delta) l} \sin \left[d \varphi_{\text {coupl }}(\delta)-\phi\right],
$$

with $\phi=d \omega_{\text {ref }}\left(L_{\text {probe }}-L_{\text {ref }}\right) / c$, where $L_{\text {probe/ref }}$ are the arm lengths of the interferometer. This off-resonant phase shift $\phi$ is adjusted to $n 2 \pi(n=0,1,2, \ldots)$ by an appropriate choice of the reference frequency $d \omega_{\text {ref }}$. In this case, the interferometer is most sensitive to phase shifts and the response is linear for small phase shifts. The mixer output signal is low pass filtered $(20 \mathrm{~Hz}, 18 \mathrm{~dB} / \mathrm{Oct})$.

The absorption of the coupling and probe field is simply obtained from the dc signals of the detectors $\mathrm{PD}_{1}$ and $\mathrm{PD}_{2}$, respectively:

$$
\begin{gathered}
I_{1}(\delta) \propto\left(E_{0}^{\text {coupl }}\right)^{2} e^{-2 \alpha_{\text {coupl }}(\delta) l}+\left(E_{0}^{\text {ref }}\right)^{2}, \\
I_{2}(\delta) \propto\left(E_{0}^{\text {probe }}\right)^{2} e^{-2 \alpha_{\text {probe }}(\delta) l} .
\end{gathered}
$$

The dc outputs of the detectors are limited to a bandwidth of $20 \mathrm{~Hz}(18 \mathrm{~dB} / \mathrm{Oct})$. The amplitude and phase signals are digitized for further digital processing and stored on a PC.

To measure a spectrum of the parametric phase shift and the coupling field absorption, the probe field is scanned \pm 50 $\mathrm{MHz}$ around the two-photon resonance while the coupling field is fixed to the $6 s_{1 / 2}, F=3 \rightarrow 6 p_{3 / 2}, F^{\prime}=4$ transition. Typical spectra are shown in Fig. 3.

We recorded spectra for coupling field powers from 1.9 $\mathrm{nW}$ to $4.2 \mathrm{~mW}$ with a fixed probe power of $140 \mu \mathrm{W}$. Each spectrum was fitted to the theoretical spectrum. From the fit, we derived the dispersion in the center and the linewidth. The linewidth was also compared to the width of the corresponding dark resonance and found to be in good agreement. The results of the fits are collected in Fig. 4. The maximum dispersion of $d \varphi_{\text {coupl }}^{\prime}=0.16 \mathrm{rad} / \mathrm{MHz}$ is achieved in the lowcoupling power regime.

Figure 5 demonstrates the good performance of the heterodyne method for $1.2-\mathrm{nW}$ and $370-\mu \mathrm{W}$ coupling and probe field intensity, respectively. The scan duration was $50 \mathrm{~s}$ and the long period noise was mainly determined by fluctuations of the atomic beam intensity.

In a second experiment, we varied the probe field power from $2.3 \mu \mathrm{W}$ to $1.1 \mathrm{~mW}$ (see Fig. 6). We obtained a maximum dispersion of $d \varphi_{\text {coupl }}^{\prime}=330 \mathrm{mrad} / \mathrm{MHz}$ at a probe power of $380 \mu \mathrm{W}$. The dispersion decreases at both higher and lower powers, in contrast to the former case, but in accor-

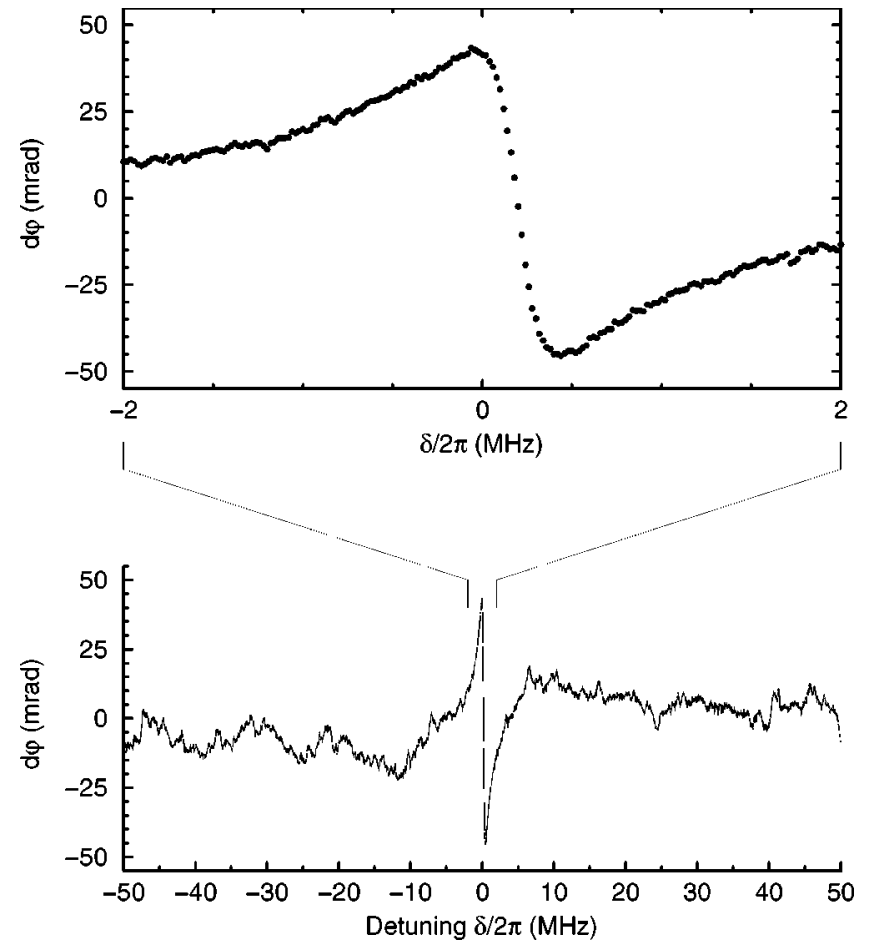

FIG. 5. Measured phase shift of the coupling beam over a span of $100 \mathrm{MHz}$ as a function of the two-photon detuning. The coupling power is $1.2 \mathrm{nW}$, the probe power is $37 \mu \mathrm{W}$, and the maximum phase shift is $88 \mathrm{mrad}$.

dance with the model. The experiment was performed at two different atomic densities. As expected, there is no influence of the density on the linewidth. Nevertheless, the dispersion shows a linear increase with the density because the sample is still optically thin.

\section{SEMICLASSICAL MODEL}

The system under investigation is well described by a simple semiclassical model, treating the atoms quantum mechanically and the fields classically. The eigenstates $|n\rangle, n$ $=a, b, c$ of the unperturbed Hamiltonian $H_{A}$ are coupled by the electric fields $E_{j}(r, t)=\operatorname{Re}\left[E_{0}^{j} \exp \left(i \omega_{j} t-\vec{k}_{j} \vec{r}\right)\right]$, where $\vec{k}_{j}$ is the wave vector for the field at frequency $\omega_{j}(j$ = probe, coupl). The coupling strength is given by the Rabi frequencies $\Omega_{a i}, i=b, c$, which are defined as $\Omega_{a b / c}$ $=\mu_{a b / c} E_{0}^{\text {probe/coupl } /(2 \hbar)}$ with the dipole matrix elements $\mu_{a b / c}$.

From the lifetime of the excited level of $30.5 \mathrm{~ns}$ [21], we derive the transition rates $\gamma_{a b}=2 \pi \times 2.18 \mathrm{MHz}$ and $\gamma_{a c}$ $=2 \pi \times 3.05 \mathrm{MHz}$. The ground-state coherence lifetime is much longer than the time the atoms spend in the interaction region because no dipole transitions are allowed and the collision rate is very low. So the effective ground-state coherence decay is equal to the transient loss rate $\gamma_{0}$, which is given by the inverse of the time $\tau$ it takes for an atom to travel through the laser field. On the other hand, the atoms are entering the interaction region with a rate $\gamma_{0}$. Therefore, 


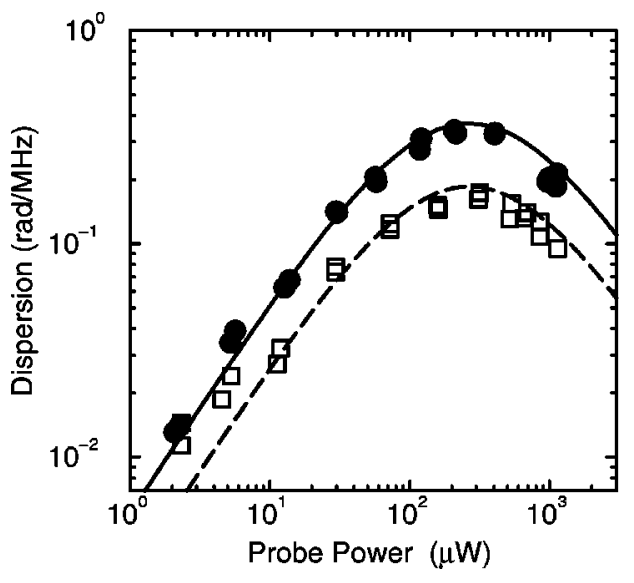

the two ground states are populated with the injection rate $\gamma_{0} / 2$.

The semiclassical master equation (A9) is given in the Appendix. The steady-state solutions of this set of coupled differential equations can be derived analytically. The simplest way to illustrate the different behavior of the parametric dispersion is by numerical simulation. In Fig. 7, typical results of a numerical simulation are shown. Graph $A$ shows the steady-state solution for the real part of $\rho_{a c}$, which corresponds to the phase shift of the coupling field. The spectrum shows a single narrow dispersive profile. In contrast to this, the real part of $\rho_{a b}$ (corresponding to the phase shift of the probe field) shows the well-known double structure (graph $C$ ) with the broad usual dispersion profile and the narrow inverted feature in the center, resulting from coherence effects. The imaginary part of both coupling and probe coherence shows similar EIT behavior, as one can see from Fig. 7, $B$ and $D$. Clearly, this is no violation of the KramersKronig relation because the parameter that is varied is the frequency of the probe laser, while the coupling laser frequency stays resonant with the coupling transition. Hence we

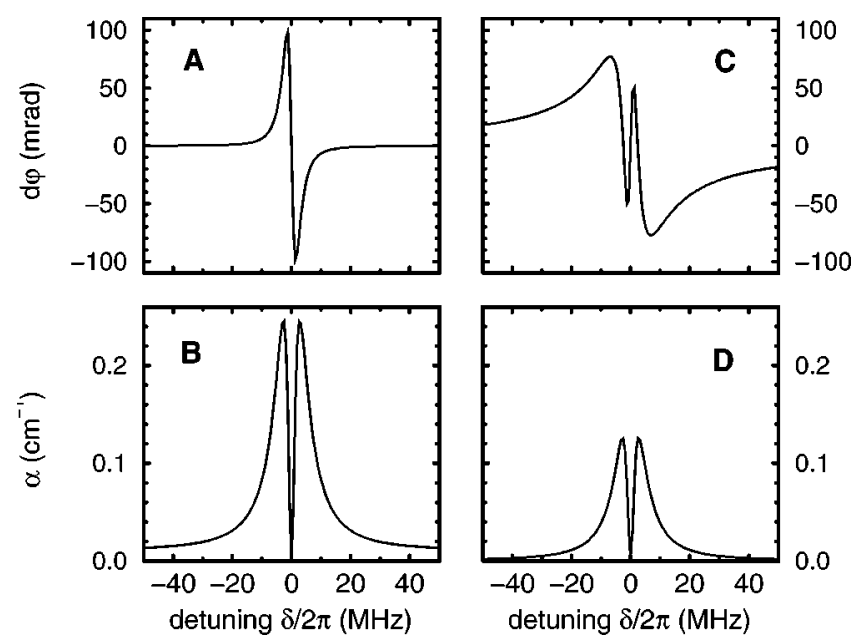

FIG. 7. Theoretical spectra of the parametric phase shift $d \varphi(A)$ and the corresponding absorption $\alpha(B)$. In $C$ and $D$, phase shift and absorption of the probe field are shown. Here $\gamma_{0}=10^{5} \mathrm{~s}^{-1}$, $\gamma_{a b}=2 \pi \times 2.18 \mathrm{MHz}, \gamma_{a c}=2 \pi \times 3.05 \mathrm{MHz}, \Omega_{a b}=\Omega_{a c}=0.3 \gamma$, $\Delta_{a c}=0$, and $N=10^{9} \mathrm{~cm}^{-3}$. call this behavior parametric dispersion.

The absorption coefficient $\alpha_{\text {coupl }}(\delta)$ and the deviation of the refractive index from unity $d n_{\text {coupl }}(\delta)$ are experimentally accessible and are related to the density-matrix elements as follows:

$$
\begin{gathered}
\frac{\operatorname{Re}\left(\rho_{a c}\right)}{\Omega_{a c}}=\frac{\hbar}{2 \pi N \mu_{a c}^{2}} d n_{\text {coupl }}(\delta), \\
\frac{\operatorname{Im}\left(\rho_{a c}\right)}{\Omega_{a c}}=\frac{\hbar c}{2 \pi N \omega_{a c} \mu_{a c}^{2}} \alpha_{\text {coupl }}(\delta)
\end{gathered}
$$

if we assume an optically thin sample with $\alpha_{\text {coupl }}(\delta) \ll l^{-1} . N$ is the atomic density and $l$ is the interaction length. The resulting phase shift is $d \varphi_{\text {coupl }}(\delta)=d n_{\text {coupl }}(\delta) \omega_{\text {coupl }} l / c$.

The dispersion of the two fields is the derivative of the real part of the matrix elements $\rho_{a c}$ and $\rho_{a b}$ with respect to the two-photon detuning $\delta$ over the corresponding Rabi frequency. The exact steady-state solutions we get from the master equation are given in the Appendix. The parametric dispersion (A10) reduces to

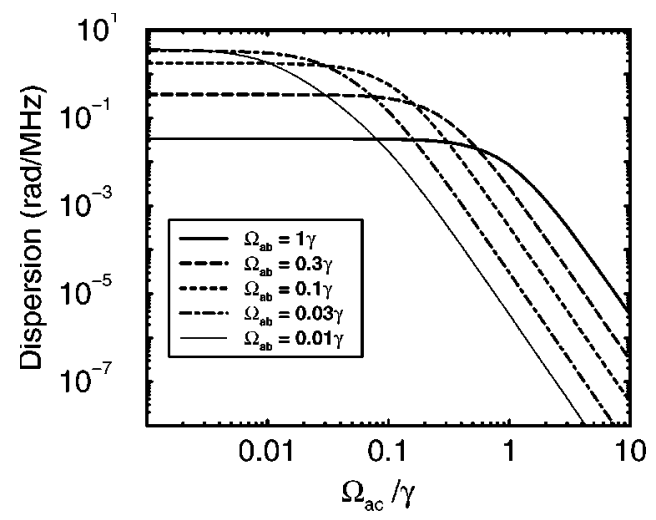

FIG. 8. Calculated absolute value of the dispersion $\varphi_{\text {coupl }}^{\prime}$ $=n_{\text {coupl }}^{\prime} \omega_{\text {coupl }} l / c$ of the coupling field for various probe field intensities with $\gamma=2 \pi \times 5.22 \mathrm{MHz}, \gamma_{0}=10^{5} \mathrm{~s}^{-1}$, and $N=10^{9} \mathrm{~cm}^{-3}$. The low power limit has a maximum of $5.5 \mathrm{rad} / \mathrm{MHz}$ at $\Omega_{a b}$ $=0.04 \gamma$. 


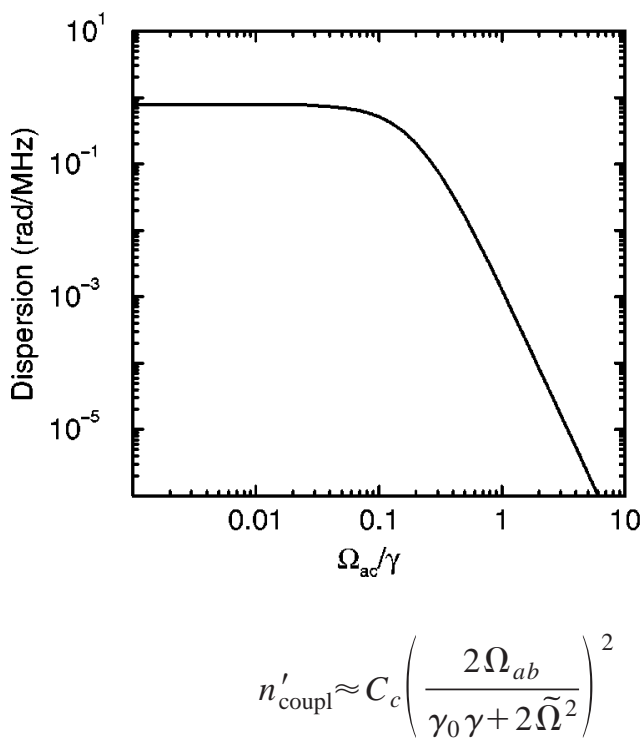

if we assume a very small decay rate of the ground-state coherence $\gamma_{0} \ll \gamma, \Omega_{a b / c}$, where $\gamma=\gamma_{a b}+\gamma_{a c}, \quad \widetilde{\Omega}^{2}=\Omega_{a b}^{2}$ $+\Omega_{a c}^{2}$, and $C_{c}=\left(2 \pi N \mu_{a c}^{2}\right) / \hbar$. The maximum achievable parametric dispersion is thus $C_{c}\left(2 \gamma_{0} \gamma\right)^{-1}$ at a probe Rabi frequency of $\Omega_{a b}=\sqrt{\gamma_{0} \gamma / 2}$, where the decay rate of the ground-state coherence $\gamma_{0}$ in our case is given by the inverse of the interaction time. In principle, the ground-state coherence can be very long-lived using cells with antirelaxation coated walls or filled with buffer gas [22,23]. For a given probe Rabi frequency, the parametric dispersion is constant for small coupling intensities. On the other side, if the coupling intensity is fixed, the parametric dispersion decreases at lower and higher probe intensities. This behavior is plotted in Fig. 8 and Fig. 9.

The probe field dispersion (A11) becomes

$$
n_{\text {probe }}^{\prime} \approx C_{p}\left(\frac{2 \Omega_{a c}}{\gamma_{0} \gamma+2 \widetilde{\Omega}^{2}}\right)^{2}
$$

for $\gamma_{0} \ll \gamma, \Omega_{a b / c}$. This quantity is a measure for the group velocity reduction of a pulse propagating through an EIT medium. The exact solution of the probe field dispersion is plotted in Fig. 10. The notches in this graph indicate a

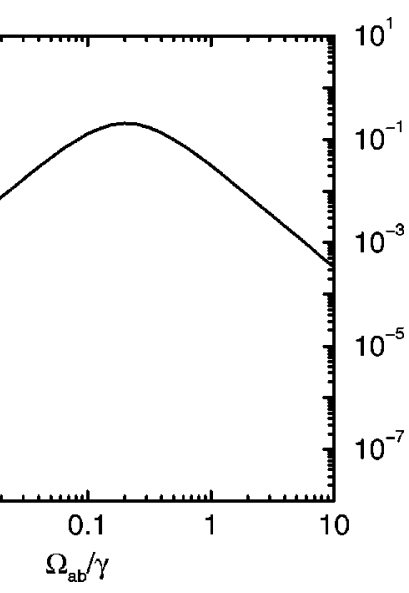

FIG. 9. Absolute value of the parametric dispersion $\varphi_{\text {coupl }}^{\prime}$ $=n_{\text {coupl }}^{\prime} \omega_{\text {coupl }} l / c$ as a function of the Rabi frequencies. $\gamma=\gamma_{a b}$ $=\gamma_{a c}=2 \pi \times 5.22 \mathrm{MHz}, \gamma_{0}=10^{5}$ $\mathrm{s}^{-1}$, and $N=10^{9} \mathrm{~cm}^{-3}$. Left: $\Omega_{a b}=0.2 \gamma$; Right: $\Omega_{a c}=0.2 \gamma$. change of sign in the dispersion. At these intensities, the dark resonance vanishes and the one-photon dispersion is left.

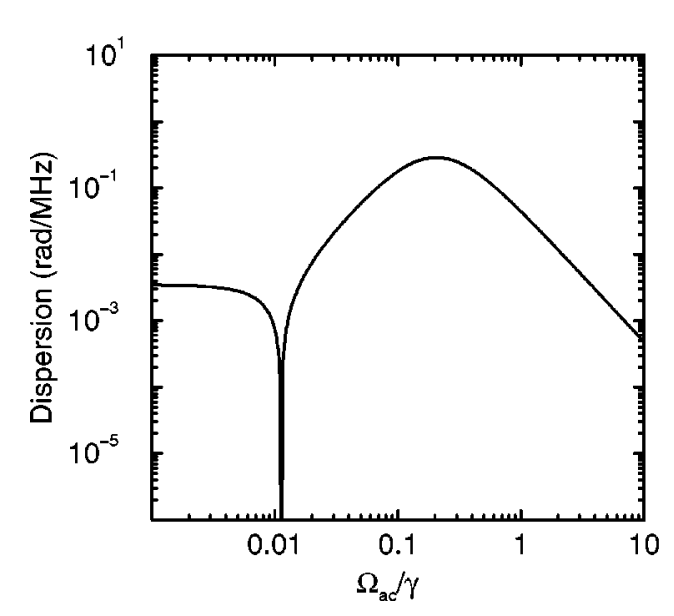

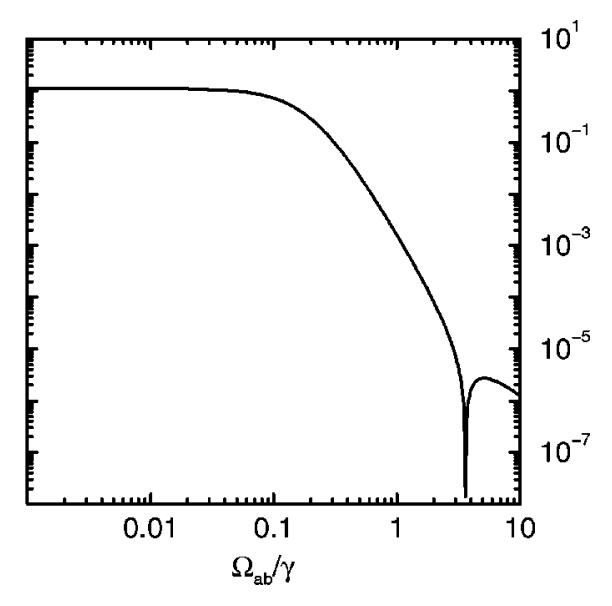

013803-6

\section{SUMMARY}

In conclusion, we have shown that the spectrum of the parametric dispersion can be reproduced with a standard semiclassical approach. We have measured the parametric phase shift and the linewidth of the EIT resonance for a wide range of probe and coupling field intensities with a new heterodyne interferometer with an off-resonant reference field. With this we achieved a significantly high signal-to-noise ratio for coupling powers down to $1.2 \mathrm{nW}$. All experimental results were found to be in good agreement with the semiclassical model.

The parametric dispersion offers the possibility to control the refractive index of a medium at a certain wavelength with a field of another wavelength. Losses are nearly completely suppressed in the vicinity of a dark resonance. By these means, the coupling beam can be deflected by an angle controlled by the probe frequency. It is therefore possible to realize an opto-optical scanner. An opto-optical phase modulator can be realized in the same way. The phase of the coupling field is controlled nearly without loss by tuning the probe field frequency.

The phase of the coupling field can also be used as an error signal for a feedback control of the difference between

FIG. 10. Absolute value of the probe field dispersion $\varphi_{\text {probe }}^{\prime}$ $=n_{\text {probe }}^{\prime} \omega_{\text {probe }} l / c$ as a function of the Rabi frequencies. The notches are roots where the dispersion changes its sign. $\gamma=\gamma_{a b}=\gamma_{a c}$ $=2 \pi \times 5.22 \mathrm{MHz}, \gamma_{0}=10^{5} \mathrm{~s}^{-1}$, $N=10^{9} \mathrm{~cm}^{-3}$. Left: $\Omega_{a b}=0.2 \gamma$; Right: $\Omega_{a c}=0.2 \gamma$. The scaling is the same as in Fig. 9. 
probe and coupling frequency. The losses in the probe power can be kept negligible in the vicinity of the dark resonance. The error signal is extremely steep because the dark resonance is much sharper than usual atomic resonances. Extending this difference frequency from the microwave into the optical regime, it is possible to close large gaps in frequency chains.

\section{ACKNOWLEDGMENTS}

This work was supported by the Sonderforschungsbereich SFB 407 of the Deutsche Forschungsgemeinschaft DFG. The authors wish to thank A. Matsko for helpful discussions.

\section{APPENDIX: ALGEBRAIC SOLUTIONS}

The dispersion can be calculated with a semiclassical model where the ensemble is treated quantum mechanically and the fields are treated classically. The detunings of the two fields from the one-photon transitions are

$$
\begin{aligned}
& \Delta_{a b}=\omega_{\text {probe }}-\left(\omega_{a}-\omega_{b}\right), \\
& \Delta_{a c}=\omega_{\text {coupl }}-\left(\omega_{a}-\omega_{c}\right),
\end{aligned}
$$

where $\Delta_{a b}$ is the probe field detuning and $\Delta_{a c}$ is the coupling field detuning from the respective atomic transition frequencies $\omega_{a}-\omega_{b}$ and $\omega_{a}-\omega_{c}$. The two-photon detuning $\delta$ is then

$$
\delta=\Delta_{a b}-\Delta_{a c} .
$$

The refractive index of the ensemble $n_{\text {coupl } / \text { probe }}=1$ $+d n_{\text {coupl/probe }}$ for the coupling and the probe laser field can be calculated from the matrix elements of the density operator $\rho$ :

$$
\begin{aligned}
& d n_{\text {coupl }}(\delta)=\frac{2 \pi N \mu_{a c}^{2}}{\hbar} \frac{\operatorname{Re}\left(\rho_{a c}\right)}{\Omega_{a c}}, \\
& d n_{\text {probe }}(\delta)=\frac{2 \pi N \mu_{a b}^{2}}{\hbar} \frac{\operatorname{Re}\left(\rho_{a b}\right)}{\Omega_{a b}} .
\end{aligned}
$$

The dispersion is defined as the derivative of the refractive index with respect to the two-photon detuning $\delta$ :

$$
n_{\text {coupl }}^{\prime}=\frac{2 \pi N \mu_{a c}^{2}}{\hbar} \frac{\partial}{\partial \delta}\left(\frac{\operatorname{Re}\left(\rho_{a c}\right)}{\Omega_{a c}}\right),
$$

$$
n_{\text {probe }}^{\prime}=\frac{2 \pi N \mu_{a b}^{2}}{\hbar} \frac{\partial}{\partial \delta}\left(\frac{\operatorname{Re}\left(\rho_{a b}\right)}{\Omega_{a b}}\right)
$$

We are now using a standard density-matrix approach (see, e.g., [24]). The dynamical behavior of the density matrix $\rho$ is given by the equation of motion (master equation)

$$
\dot{\rho}=-\frac{i}{\hbar}\left[H_{A}-\vec{\mu} \vec{E}, \rho\right]+\text { relaxation terms, }
$$

where $\hat{\mu}$ is the electrical dipole operator and $\vec{E}$ is the sum of the two electrical fields. The relaxation terms are added in a phenomenological way (see [25], and references therein).

With a transformation into the interaction picture and neglecting the fast oscillating terms (rotating-wave approximation), the master equation becomes

$$
\begin{gathered}
\dot{\rho}_{c c}=\gamma_{0} / 2-\gamma_{0} \rho_{c c}+\gamma_{a c} \rho_{a a}+i\left(\Omega_{a c} \rho_{c a}-\Omega_{c a} \rho_{a c}\right), \\
\dot{\rho}_{b b}=\gamma_{0} / 2-\gamma_{0} \rho_{b b}+\gamma_{a b} \rho_{a a}+i\left(\Omega_{a b} \rho_{b a}-\Omega_{b a} \rho_{a b}\right), \\
\dot{\rho}_{a a}=-\left(\gamma_{0}+\gamma_{a b}+\gamma_{a c}\right) \rho_{a a}-i\left(\Omega_{a b} \rho_{b a}-\Omega_{b a} \rho_{a b}\right) \\
-i\left(\Omega_{a c} \rho_{c a}-\Omega_{c a} \rho_{a c}\right), \\
\dot{\rho}_{a b}=-\Gamma_{a b} \rho_{a b}-i \Omega_{a b}\left(\rho_{b b}-\rho_{a a}\right)-i \Omega_{a c} \rho_{c b}, \\
\dot{\rho}_{a c}=-\Gamma_{a c} \rho_{a c}-i \Omega_{a c}\left(\rho_{c c}-\rho_{a a}\right)-i \Omega_{a b} \rho_{b c}, \\
\dot{\rho}_{b c}=-\Gamma_{b c} \rho_{c b}+i \Omega_{a c} \rho_{b a}-i \Omega_{b a} \rho_{a c} .
\end{gathered}
$$

The decay rates are

$$
\begin{gathered}
\Gamma_{a b}=\frac{1}{2}\left(2 \gamma_{0}+\gamma_{a b}+\gamma_{a c}\right)+i \Delta_{a b}, \\
\Gamma_{a c}=\frac{1}{2}\left(2 \gamma_{0}+\gamma_{a b}+\gamma_{a c}\right)+i \Delta_{a c}, \\
\Gamma_{b c}=\gamma_{0}-i \delta .
\end{gathered}
$$

Setting the time derivatives to zero, $\dot{\rho}=0$, we obtain the steady-state equations. This set of coupled linear equations can be solved analytically. We assume the coupling field to be on resonance $\left(\Delta_{a c}=0, \Delta_{a b}=\delta\right)$. Without any further assumptions, the steady-state solution of the master equation (A9) yields the following expression for the dispersion of the coupling field at the two-photon resonance:

$$
\frac{\partial}{\partial \delta}\left(\frac{\operatorname{Re}\left(\rho_{a c}\right)}{\Omega_{a c}}\right)=\frac{4 \Omega_{a c}^{2}\left\{\gamma_{0}\left(\gamma+\gamma_{0}\right)\left(\gamma+2 \gamma_{0}\right)\left(\gamma+3 \gamma_{0}\right)+\left[4 \gamma_{a c}\left(\gamma+4 \gamma_{0}\right)+6 \gamma_{0}^{2}\right] \Omega_{a b}^{2}+\left[4\left(\gamma_{a b}+\gamma_{0}\right)\left(\gamma+2 \gamma_{0}\right)-2 \gamma_{0}^{2}\right] \Omega_{a c}^{2}\right\}}{\left(2 \gamma_{0}+\gamma\right)\left[2 \gamma_{0}^{2}+\gamma \gamma_{0}+2 \widetilde{\Omega}^{2}\right]^{2}\left[\gamma_{0}\left(\gamma+\gamma_{0}\right)\left(\gamma+2 \gamma_{0}\right)+\left(4 \gamma_{a c}+8 \gamma_{0}\right) \Omega_{a b}^{2}+\left(4 \gamma_{a b}+8 \gamma_{0}\right) \Omega_{a c}^{2}\right]} .
$$

The dispersion of the probe field becomes 


$$
\begin{aligned}
\frac{\partial}{\partial \delta}\left(\frac{\operatorname{Re}\left(\rho_{a b}\right)}{\Omega_{a b}}\right) & \\
= & \frac{2 \gamma_{0}\left(\gamma+\gamma_{0}\right)\left[\gamma_{0}\left(\gamma+2 \gamma_{0}\right)+2 \Omega_{a b}^{2}\right]^{2}}{\left(2 \gamma_{0}+\gamma\right)\left[2 \gamma_{0}^{2}+\gamma \gamma_{0}+2 \widetilde{\Omega}^{2}\right]^{2}\left[\gamma_{0}\left(\gamma+\gamma_{0}\right)\left(\gamma+2 \gamma_{0}\right)+\left(4 \gamma_{a c}+8 \gamma_{0}\right) \Omega_{a b}^{2}+\left(4 \gamma_{a b}+8 \gamma_{0}\right) \Omega_{a c}^{2}\right]} \\
& -\frac{4\left\{\gamma_{0}\left(\gamma+2 \gamma_{0}\right)\left(\gamma^{2}+\gamma_{0}^{2}+4 \gamma_{0} \gamma_{a c}\right)+2\left[\gamma_{0}^{2}-2 \gamma_{0}\left(\gamma_{a b}-3 \gamma_{a c}\right)+2 \gamma \gamma_{a c}\right] \Omega_{a b}^{2}\right\} \Omega_{a c}^{2}-8\left(\gamma+2 \gamma_{0}\right)\left(\gamma_{0}+2 \gamma_{a b}\right) \Omega_{a c}^{4}}{\left(2 \gamma_{0}+\gamma\right)\left[2 \gamma_{0}^{2}+\gamma \gamma_{0}+2 \widetilde{\Omega}^{2}\right]^{2}\left[\gamma_{0}\left(\gamma+\gamma_{0}\right)\left(\gamma+2 \gamma_{0}\right)+\left(4 \gamma_{a c}+8 \gamma_{0}\right) \Omega_{a b}^{2}+\left(4 \gamma_{a b}+8 \gamma_{0}\right) \Omega_{a c}^{2}\right]}
\end{aligned}
$$

with $\widetilde{\Omega}^{2}=\Omega_{a b}^{2}+\Omega_{a c}^{2}$ and $\gamma=\gamma_{a b}+\gamma_{a c}$.

[1] S.E. Harris, Phys. Today 50 (7), 36 (1997).

[2] B.S. Ham et al., Opt. Commun. 144, 227 (1997).

[3] A.S. Zibrov et al., Phys. Rev. Lett. 76, 3935 (1996).

[4] H. Schmidt et al., Opt. Lett. 21, 1936 (1992).

[5] A. Wicht et al., Opt. Commun. 134, 431 (1997).

[6] A. Wicht et al., Opt. Commun. 179, 107 (2000).

[7] L.J. Wang, A. Kurzmich, and A. Dogario, Nature (London) 406, 277 (2000).

[8] L.V. Hau, S.E. Harris, Z. Dutton, and C.H. Behroozi, Nature (London) 397, 594 (1999).

[9] A. Imamoḡlu, Opt. Commun. 179, 179 (2000).

[10] A. Matsko et al., Phys. Rev. Lett. 84, 5752 (2000).

[11] M. Fleischhauer and M.O. Scully, Phys. Rev. A 49, 1973 (1994).

[12] R. Wynands and A. Nagel, Appl. Phys. B: Lasers Opt. 68, 1 (1999).

[13] G. Müller et al., Phys. Rev. A 56, 2385 (1997).

[14] G. Alzetta et al., Nuovo Cimento Soc. Ital. Fis., B 36, 5
(1976).

[15] M. Xiao, Y. Li, S. Jin, and J. Gea-Banacloche, Phys. Rev. Lett. 74, 666 (1995).

[16] G. Müller, A. Wicht, R.-H. Rinkleff, and K. Danzmann, Opt. Commun. 127, 37 (1996).

[17] M. Müller et al., Phys. Rev. A 62, 060501 (2000).

[18] A. Wicht et al., Appl. Phys. B: Lasers Opt. 70, 821 (2000).

[19] J.L. Hall, Ma Long-Sheng, and G. Kramer, IEEE J. Quantum Electron. 23, 427 (1987).

[20] R.D. Swenumson et al., Rev. Sci. Instrum. 52, 559 (1981).

[21] R.J. Rafac et al., Phys. Rev. A 50, R1976 (1994).

[22] D. Budker, V. Yashchuk, and M. Zolotorev, Phys. Rev. Lett. 81, 5788 (1998).

[23] M.M. Kash et al., Phys. Rev. Lett. 82, 5229 (1999).

[24] G. Orriols, Nuovo Cimento Soc. Ital. Fis., B 53, 1 (1979).

[25] E. Arimondo, in Progress in Optics, edited by E. Wolf (Elsevier, Amsterdam, 1996), Vol. 35, pp. 257-354. 